\title{
Tendon structure, disease, and imaging
}

\author{
Jeffrey $\mathrm{H}$. Weinreb \\ Chirag Sheth \\ John Apostolakos \\ Mary-Beth McCarthy \\ Benjamin Barden \\ Mark P. Cote \\ Augustus D. Mazzocca
}

Department of Orthopaedic Surgery, UCONN Health Center, Farmington, USA

Corresponding author:

Augustus D. Mazzocca

Department of Orthopaedic Surgery

UCONN Health Center

Farmington, CT 06034, USA

E-mail: mazzocca@uchc.edu

\section{Summary}

Tendon imaging plays a critical role in evaluating tendon diseases and injuries including mechanical, degenerative, and overuse disease, inflammatory enthesitis, as well as partial and full thickness tears. Ultrasound and magnetic resonance imaging (MRI), each with unique benefits and limitations, are commonly utilized to assist in diagnosing these diseases and conditions. This review delineates important structural properties of tendon and biochemical changes occurring in tendon pathology. This review also examines commonly injured tendons including tendons of the elbow, tendons of the rotator cuff of the shoulder, hip abductor tendons, patellar tendons, and the Achilles tendon to help clinicians better recognize tendon disease. Finally, this paper introduces several emerging imaging techniques including $\mathrm{T}_{2}$ mapping, ultra-short echo time MRI, and sonoelastography as ways in which tendon imaging and evaluation may be improved.

KEY WORDS: imaging, Magnetic Resonance Imaging, tendinopathy, ultrasound.

\section{Background}

Tendons are ubiquitous, dense, regular connective tissue structures in the body and are defined by their anatomical position connecting muscle to bone ${ }^{1}$. Ten- dons consist of cross-linked triple helices of mostly type I collagen and enable the transfer of mechanical load generated by a muscle to bone, resulting in stabilization or movement across a joint ${ }^{1,2}$. Tendon matrix composition and structure vary considerably based on functional requirements and body position ${ }^{1}$. Changes to tendon composition predispose tendinopathy, which includes mechanical, degenerative, and overuse disease, as well as inflammation of the entheses. Tendons may also be fully or partially torn following tendinipathy or trauma. Several advanced imaging modalities play a critical role in comparingnormal and abnormal tendon structures. Magnetic resonance imaging (MRI) and ultrasound (US) play a critical role in comparing normal and abnormal tendon structure and in the evaluation of tendon disease $^{2}$. However, new modalities including $T_{2}$ mapping, ultra-short echo time MRI, and sonoelastography are emerging as candidates for improving tendon disease imaging.

\section{Normal Tendon Structure}

The dry weight of normal tendons generally consists of $65-80 \%$ collagen (mostly type I), and includes $1-2 \%$ elastin embedded in a proteoglycan-water matrix ${ }^{3}$. The remainder of the collagen structure includes other types of collagen including type II in cartilaginous zones, types III, IV, and V in the vasculature, and type $X$ in the mineralized fibrocartilage near entheses, among others ${ }^{4,5}$. The remainder of the tendon structure consists of tenocytes and tenoblasts between collagen fibers and ground substance surrounding the collagen including proteoglycans, glycosaminoglycans, structural glycoproteins, and other small molecules $^{3}$. Inorganic components from less than $0.2 \%$ of dry tendon mass, with calcium representing the most abundant inorganic tendon component ${ }^{4}$. There is increased calcium concentration at the insertion and decreased concentration in the tensional area.

Individual collagen fibrils are organized into collagen fibers, which are subsequently organized into subfasicles. An endotendon sheath encloses subfasicles and fascicles. The number of collagen fibers in each subfasicle may vary considerably from tendon to tendon ${ }^{3}$. A group of fascicles is organized into tertiary fiber bundles, which are grouped together and enclosed by the epitendon to form a tendon ${ }^{3}$. Fiber orientation varies depending on location and functional requirements. Collagen fibers are arranged parallel to the axis of the tendon when force is directed along this axis, as in the patellar tendon ${ }^{2}$. Complex fiber patterns, including plait 
formation, crossing, and up-tying fibers have been described $^{3}$. The tendon structure is further complicated when multiple muscles converge on a single tendon, as in the Achilles or quadriceps tendon ${ }^{2}$.

\section{Tendon Pathology}

There are two general classifications of tendon disease. Inflammatory enthesitis refers to inflammation of the entheses while tendinopathy refers to mechanical, degenerative, and overuse disease ${ }^{2}$.

\section{Inflammatory Enthesitis}

The entheses are the sites of attachment of tendon, ligament, fascia, or joint capsule to bone. The argument has been made that any fibrocartilagineous structures contributing to this role can be considered part of the 'enthesis organ' and may include the enthesis, seasamoid, and periosteal fibrocartilage as well as associated bursa and synovial fluid ${ }^{6}$. Inflammatory enthesitis occurs when this area becomes inflammed and is a distinguishing pathological feature of spondyloarthritides ${ }^{6}$. Inflammatory enthesitis may be either painful or asymptomatic and may be visualized as soft tissue swelling. Although obtaining enthesis tissue proves difficult, histological changes including macrophage mediated fibrocartilage destruction, lymphocytic infiltration within bone, lymphocyte paucity at the insertion site, and macrophage infiltration have been reported 6,7 .

\section{Tendinopathy}

Historically, pain associated with tendon overuse is referred to as tendinitis. Although the etymology of tendinitis suggests an inflammatory process, research indicates tendons exposed to overuse demonstrate little or no inflammation ${ }^{8}$. Tendinopathy has therefore been supported as a classification of the painful condition occurring in and around tendons accompanying mechanical, degenerative, and overuse disease. Tendinopathy is associated with degeneration and disorganization of the collagen structure and an increase in mucoid, proteoglycan and water content ${ }^{2,8-12}$. A 10-20 fold calcium concentration increase may also occur ${ }^{3}$. Chemical and molecular changes, including matrix metalloproteinase production, tendon cell apoptosis, chondroid metaplasia of the tendon, and expression of protective factors including insulinlike growth factor 1 and nitric oxide synthetase, have been described in tendon overuse ${ }^{8}$. Macroscopic changes include tendon thickening, loss of mechanical properties, and pain 2,8,9,12. Collagen fibril rupture may occur and progress to full-thickness tears. These degenerative changes generally precede a full thickness tear, which does not typically occur in a healthy tendon ${ }^{2}$. Following a tear, there may be ingrowth of vessels into the tendon.
Aging tendons undergo structural and compositional changes including decreased water content and collagenous changes. These changes may predispose both tendinopathy and inflammatory enthesitis ${ }^{13}$. Tendon disease more commonly occurs in hypovascular segments of tendon. Decreased tendon vascularity is associated with aging 2,14 . These changes play an important role in tendon imaging.

\section{Imaging Techniques}

\section{Magnetic Resonance Imaging}

MRI is widely used to evaluate tendon disease. MRI is an attractive option as it is non invasive and does not utilize ionizing radiation. Patients with implanted devices have historically been unable to undergo MRI, but as technology improves, MRI is contraindicated in fewer patients ${ }^{15}$. Tendon structure plays a critical role in determining appearance in MRI. The water and collagen in tendon are aligned, shortening the $T_{2}$ relaxation time to $1-2 m s^{16-18}$. The $T_{1}$ relaxation time is relatively short at approximately $600 \mathrm{~ms}$ at 3 $\mathrm{T}^{2}$. With conventional MRI techniques, these strong dipole interactions may result in a dark signal void. However, if tendons are examined at $55^{\circ}$ to the static magnetic field, this signal becomes detectable. This angle is known colloquially as the magic angle ${ }^{18-20}$. $\mathrm{T}_{2}$-weighted imaging sequences have beenshown to be more dependent on fiber orientation than $T_{1}{ }^{21}$. As previously discussed, the orientation of tendon fibers may change along its course, resulting in magic angle distortional effects. This problem may be mitigated by imaging with a sufficiently long echo time ${ }^{2}$. A stronger magnetic field may also be useful in clarifying the signal noise from tendon by improving image resolution and increasing the signal-to-noise ratio 22 .

An increase in signal intensity on T2 weighted imaging sequences is often the first sign of tendon abnormality on MRI. Thickening of the tendon may also be seen $^{2}$. MRI of inflammatory enthesitis demonstrates tendon enlargement, loss of the normal flattened hypointense (dark) appearance, focal thickening, and rounded configuration at the insertion site 23 .

Although the appearance of a full thickness or partial tear is variable, a hyperintense (white) signal may be seen at the site of the tear or in the surrounding tissue (Fig. 1). Complete rupture of the tendon and displacement of adjacent features may also be seen (Fig. 2). Scarring can obscure this effect and result in an intermediate signal ${ }^{2}$. Fatty tissue infiltration has also been reported following a tendon tear ${ }^{24}$. Fatty tissue may be differentiated from fluid on $T_{2}$ weighted $\mathrm{MRI}$ as fluid appears darker than fatty tissue ${ }^{25}$.

\section{Ultrasound}

Much like MRI, US imaging does not utilize ionizing radiations or require an invasive procedure. US examination has the added advantage of allowing ten- 


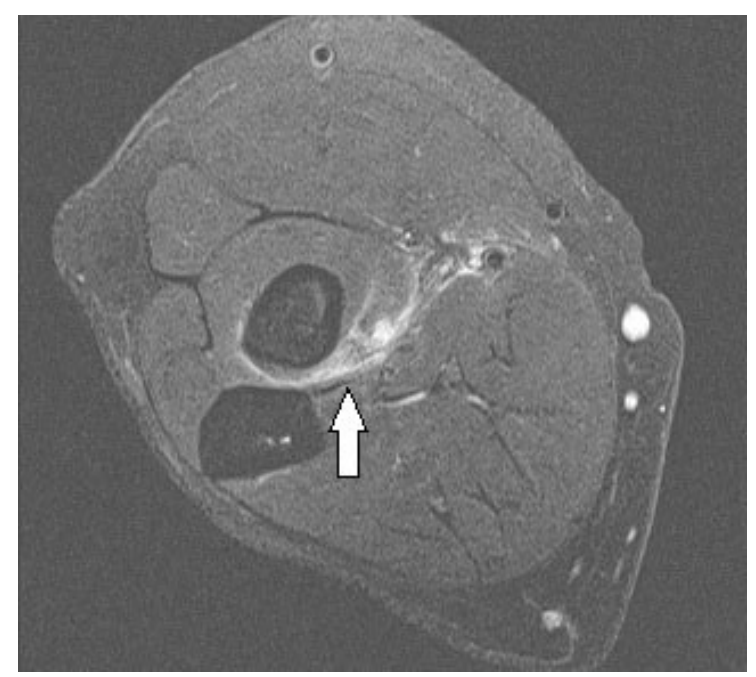

Figure 1. Axial proton-density weighted MRI of severe insertional bicep tendon partial-thickness tear with tendinopathy.

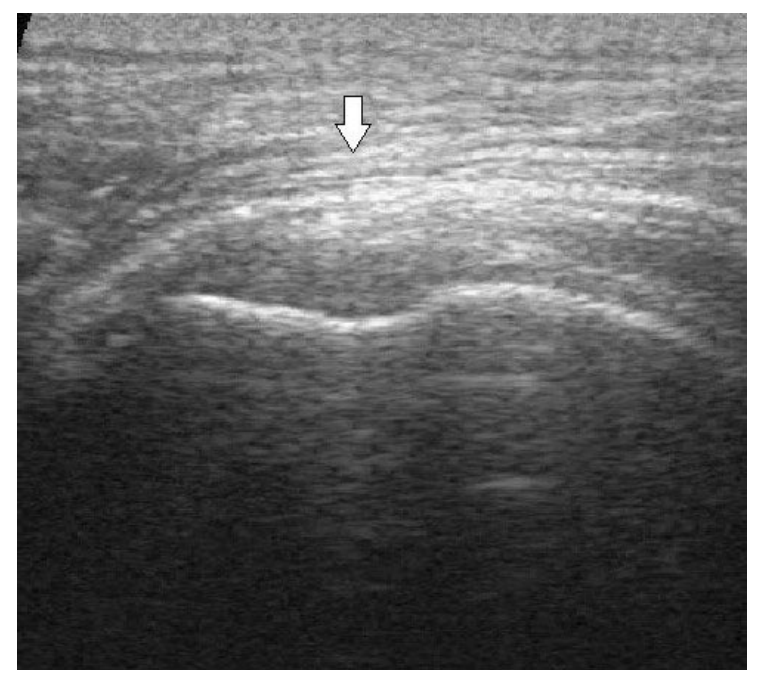

Figure 3. Longitudinal ultrasound of an intact supraspinatus tendon one year after successful repair. The arrow demonstrates the parallel echogenic lines representing the fascicles of the tendon.

don imaging in a weight bearing position and from many angles ${ }^{2}$. However, US is unable to image through bone or gas-containing structures, making visualization of articular or deep-lying tendons difficult. When viewed with a high-frequency transducer, a longitudinal orientation reveals parallel echogenic lines representing the fascicles of a tendon (Fig. 3). Viewed axially, the fascicles appear as echogenic points or lines ${ }^{2}$. Ideal frequency for evaluation depends on tendon depth.

US examination can visualize disruptions in the tendon fascicular pattern.Pathologic changes may include increased space between echogenic fibers and

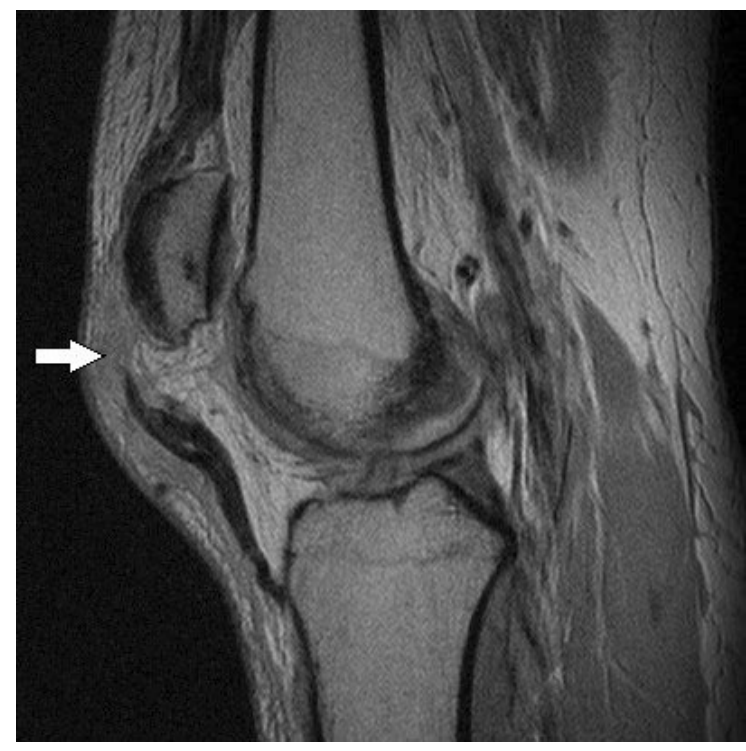

Figure 2. Sagittal proton-density-weighted turbo spin-echo MRI of a full thickness patellar tear with patella alta.

decreased echogenicity. Similar to MRI, diseased tendon may appear thicker on US². US of inflammatory enthesitis may show loss of normal fibrillarechotexture, blurring of tendon margins, and irregular fusiform thickening ${ }^{23}$. Calcification that may occur in tendinopathy can be visualized as bright echogenic regions with US and neovascularization occurring in a tear may be visualized with Power Doppler US ${ }^{26}$. The imaging timescale is crucial in US as the anechoic fluid buildup that follows an acute tear eventually becomes organized and is difficult to distinguish from associated tendon².

\section{Common Tendon Injuries, Imaging, and Ac- curacy}

\section{Medial and Lateral Epicondylitis}

Lateral and medial epicondylitis, known as 'tennis elbow' and 'golfer's elbow' respectively, are most commonly seen resulting from repetitive stress ${ }^{27}$. Lateral epicondylitis occurs when repeated pronation and supination occur in an extended elbow position involving the common extensor tendon while medial epicondylitis occurs following repetitive throwing motions and typically involves the pronator teres and flexor carpi radialis near their condylar insertion². Lateral epicondylitis occurs seven to 10 times more frequently than medial epicondylitis ${ }^{27}$. On US, medial and lateral epicondylitis appear as tendon enlargement and heterogeneity. Tears may be visualized as hypoechoic regions with adjacent discontinuity. Calcific changes may also be visualized ${ }^{27}$. MRI findings of tendinopathy for $T_{1}$ and $T_{2}$ weighted imaging include intermediate signal intensitywithin the substance of the tendon with or without tendon thicken- 
ing ${ }^{27}$. Partial thickness tears can be visualized as regions of fluid signal intensity within the tendon with tendon thinning while full-thickness tears appear as a fluid filled gap across the substance of the tendon²,27. A fluid signal may also be seen adjacent to both full and partial thickness tears ${ }^{2}$.

In a study by Miller et al. the sensitivity of US for the detection of both lateral and medial epicondylitis ranged from 64 to $82 \%$, while the sensitivity of MRI was between 90 and $100 \%$ indicating careful selection of imaging technique and operator is important when detecting epicondylitis ${ }^{28}$.

\section{Rotator Cuff of the Shoulder}

The term "rotator cuff" describes the tendons connecting the infraspinatus, supraspinatus, teres minor, and subscapularis muscles to the humeral head.Traumatic tears of the rotator cuff tend to occur at the tendonbone junction of the supraspinatus and greater tuberosity of the humerus whereas degenerative tears tend to be seen posteriorly at the junction of the supraspinatus and infraspinatus ${ }^{2,29}$. Recent evidence strongly suggests that most rotator cuff tears are caused by primary intrinsic degeneration ${ }^{30}$. Tears of the subscapularis tendon may occur in isolation, however, concomitant tears of the supraspinatus or infraspinatus tendons are more common. Teres minor tendon tears are rare ${ }^{31}$. Partial tears are more common than full thickness tears and tears are more commonly located on the articular surface compared to bursal or intrasubstance locations ${ }^{32}$.

On MRI, partial tears appear with low/intermediate signal on $T_{1}$ weighted images and may be difficult to differentiate from tendinosis ${ }^{2}$. $T_{2}$ weighted imaging with fat suppression results in a high signal intensity and is most useful for diagnosing tears ${ }^{33}$. The tendon surface may also present with thickening or thinning. Additional signs of a rotator cuff tear include fluid filled defects in the tendon superstructure and fluid superiorly, anteriorly, or inferiorly to a supraspinatus tear. Tendon retraction may or may not occur (Fig. 4) ${ }^{2}$. Hyperdense calcification and bursitis may also be present (Fig. 5) ${ }^{34}$. On US, full thickness tears may present as focal defects, however, visualization of the tendon may be difficult or impossible if retraction has occurred (Fig. 6). Partial thickness rotator cuff tears may be hypoechoic or contain heterogenic echogenicity tendon signals which may be also accompanied by structural flattening of the bursal surface 2,35 .

A recent meta-analysis examining 65 rotator cuff tear studies in which full and partial thickness tears were evaluated determined the sensitivity for diagnosing a full thickness tear with MRI and US to be $92.1 \%$ and $92.3 \%$ respectively. The specificity for diagnosing a full thickness tear on MRI and US is $92.9 \%$ and $94.4 \%$ respectively. This sensitivity of MRI and US for diagnosing a partial thickness tear is considerably lower than a full thickness tear at $63.6 \%$ and $66.7 \%$ respectively. The specificity for diagnosing a partial tear in $\mathrm{MRI}$ and $\mathrm{US}$ is $91.7 \%$ and $93.5 \%$ respectively ${ }^{36}$. These data suggest that MR and US are comparable in diagnosing full and partial thickness rotator cuff tears.

The accuracy of detecting tendinopathy on MRI and US has also been described. The sensitivity of MRI and US was found to be $55 \%$ and $66.7 \%$ respectively while the specificity was determined to be $92.7 \%$ and $88.4 \%$ respectively. Although these data suggest US may be more sensitive and less specific than MRI, more high quality studies are needed.

\section{Gluteal Tendons}

The gluteus medius and gluteus minimus tendons insert on the greater trochanter of the hip and have been referred to as "the rotator cuff of the hip". Both tendons have been implicated in Greater Trochanteric Pain Syndrome (GTPS) 37,38 . GTPS may mimic other serious conditions including a vascular necrosis and stress fracture.Therefore, accurate imaging and evaluation of these tendinous structrures is critical to obtaining an accurate diagnosis ${ }^{39}$. On MRI, gluteal tendinopathy may be seen as tendon thickening or increased tendon signal intensity on T2-weighted images without tendon discontinuity or thinning.

MRI permits tendon tear visualization as well. Tendon thinning on $T_{1}$ and $T_{2}$ weighted images in conjunction

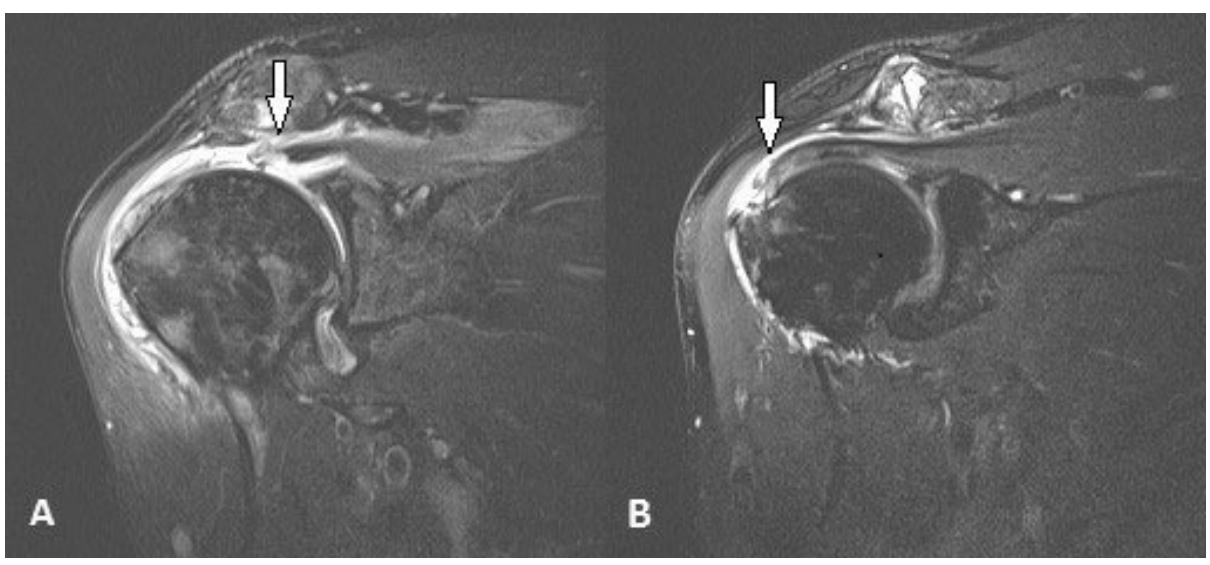

Figure 4. A) Coronal $T_{2}$ weighted MRI of rotator cuff demonstrating full thickness supraspinatus tendon tear with retraction to the glenoid. B) Coronal $\mathrm{T}_{2}$ weighted MRI of the rotator cuff demonstrating a full thickness supraspinatus tendon tear without retraction. 


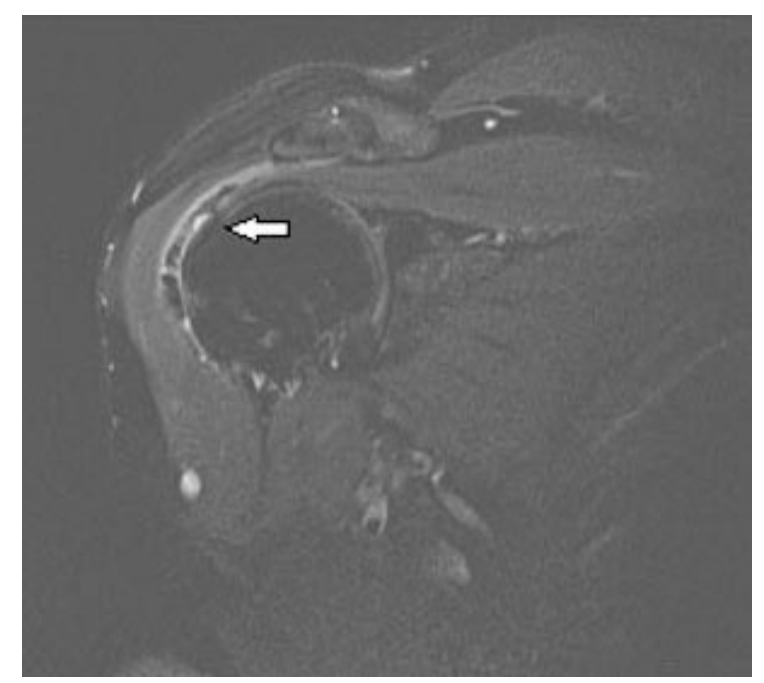

Figure 5. Coronal $\mathrm{T}_{2}$ weighted MRI of supraspinatus tendiosius with reactive subacromial subdeltoid bursitis, intrasubstance tear, and hydroxyapatite calcification.

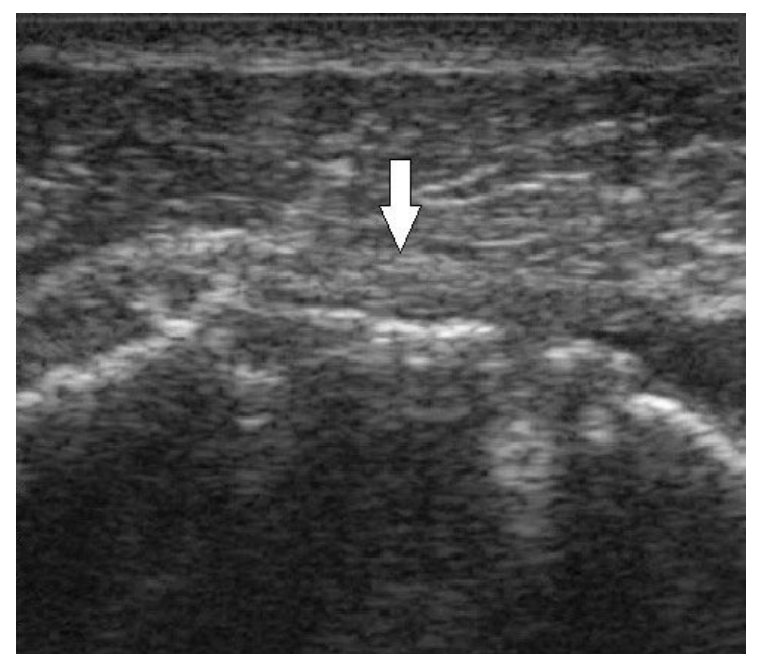

Figure 6. Longitudinal ultrasound of a torn supraspinatus tendon three months after surgery. Due to retraction, the echogenic tendon fascicles cannot be visualized. Arrow indicates where the tendon would normally be visualized.

with high signal intensity in the tendon on $\mathrm{T}_{2}$ weighted sequences is indicative of a partial thickness tear. Discontinuity of tendon fibers with a high signal intensity on T2-weighted images are suggestive of full thickness tears ${ }^{38}$. MRI may also reveal bursal fluid and hypointensity caused by calcification in areas of tendinopathy 2 . Using US, gluteal tendinosis may present as decreased or heterogeneous echogenicity, often with tendon thickening. Tendon tears may manifest as tendon thinning or as anechoic defects within the tendon ${ }^{40}$. Calcification may appear as hyperechogenic foci in the tendon².

A systemic review by Westacott et al. reported that MRI had a sensitivity of $33-100 \%$ and a specificity of $92-100 \%$ when detecting gluteal tendon tears. In the same review, US had a sensitivity of $79-100 \%$ and positive predictive value of $95-100 \%{ }^{37}$. This data suggests that US may be the preferred imaging technique, although additional well-designed studies are required. Additionally, the sensitivity and specificity of US to detect tendinous pathology is highly dependent on the administering technician ${ }^{37}$.

\section{Patellar Tendon}

Patellar tendon disease is associated with instability and over use, especially accompanying jumping sports and is seen in the deep, medial, or central part of the tendon near its insertion ${ }^{2}$. Normal tendon appears with low signal intensity on MRI, although the patellar enthesis may be hyperintense (white) and US demonstrates the typical echoic fibrillar structure ${ }^{41}$. In tendon disease, US demonstrates disruption of this fibrillar structure, hypoechogenicity, and swelling ${ }^{2}$. However, it should be noted that a hypoechoic signal may be seen in asymptomatic patients and clinical assessment is important in the appropriate management of patellar tendon disease ${ }^{42}$. On MRI, focal thickening of the tendon may be seen in conjunction with increased signal intensity ${ }^{2}$. In cases of complete tendon rupture, the tendon appears discontinuous on MRI and may be accompanied by patella alta (Fig. 2). In a study by Warden et al., MRI and US had equivalent specificity (82\%) for detecting patellar. Tendinopathy while the sensitivity of US was greater than that of MRI and $87 \%$ and $57 \%$ respectively 43,44 .

\section{Achilles Tendon}

The combined insertions of the soleus and gastrocnemis muscles on the calcaneus tuberosity form the Achilles tendon. The Achilles tendon produces a low signal on all conventional MRI sequences ${ }^{2,45}$. On US, normal Achilles tendon appears as a ribbon-like, hypoechoic, tightly packed fibrillarstructure ${ }^{2,46}$. In tendonopathy, the tendon may appear thickened with convexity at the anterior border ${ }^{47}$. MRI may reveal an intermediate signal in $T_{1}$ weighted images, and a high signal in or around the tendon in fluid-sensitive MRI sequences, especially in the retrocalcaneal bursa, or as bony edema (Fig. 7) ${ }^{2}$. If the tendon has been completely ruptured, a hyperdense fluid signal may be seen in the tendon space (Fig. 8). US may reveal hypoechogenicity with separation of the fibrillar structure or neovascularization by Doppler flow ${ }^{2}$. Additionally, mechanical tendon disease, visualized as microtearing on US in asymptomatic patients, generally affects the mid-section of a tendon ${ }^{48}$. Obesity has been shown to be an important risk factor for Achilles tendinopathy. Running is also associated with physiologic hypertrophy of the Achilles tendon and overweight runners may precociously develop tendon abnormalities ${ }^{49}$.

Although US has been shown to be both sensitive and specific for diagnosing partial and full-thickness 


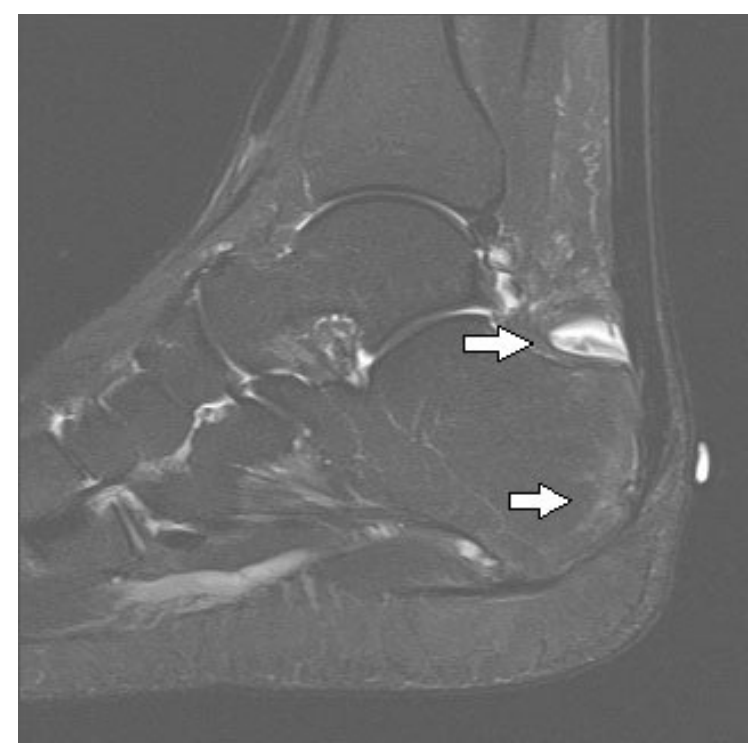

Figure 7. Sagittal $T_{2}$ weight edinsertional Achilles tendinopathy with reactive boney edema pattern (lower arrow) in the calcaneus and retrocalcaneal bursitis (upper arrow).

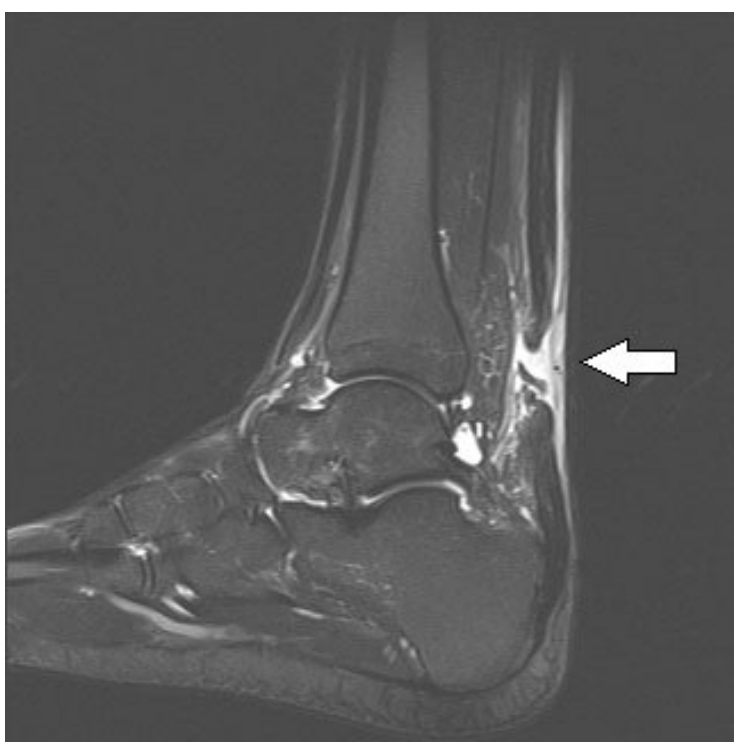

Figure 8. Sagittal $\mathrm{T}_{2}$ weighted MRI of the Achilles tendon demonstrating a full thickness tear with fluid noted by arrow.

Achilles tendon tears, MRI is believed to be more sensitive for detecting partial tears based on studies comparing the sensitivity of US and MRI ${ }^{11,50}$.

\section{Emerging Techniques}

\section{Ultrashort Echo Time MRI}

In conventional MRI one is limited when it comes to imaging tissues with a $T_{2}$ signal that is significantly shorter than 10 microseconds $^{2}$. Using ultrashort echo time MRI techniques, it is possible to acquire images with echo times as low as a few microseconds. This technique will allow new options to characterize these tissues, including tendon entheses ${ }^{51}$.

\section{Sonoelastography}

Sonoelastography estimates the hardness or softness of a tissue by measuring the strain caused by tissue compression ${ }^{52}$. It is a noninvasive estimation of tissue stiffness based upon the fact that soft tissue has greater tissue displacement than hard tissue when externally compressed ${ }^{53}$. This is useful when disease occurs, as there are changes to the biomechanical properties of tendon ${ }^{54}$. Sonoelastography allows calculation and comparison of tissue displacement before and after tissue compression with conventional US equipment but modified software, making widespread implementation more convenient ${ }^{55}$.

\section{$T_{2}$ Mapping}

$T_{2}$ mapping was developed to exploit the sensitivity of $\mathrm{MRI}$ to the biophysical properties of cartilage ${ }^{56}$. Unlike anatomic imaging, $\mathrm{T}_{2}$ mapping is sensitive to changes in the chemical composition and structure of cartilage ${ }^{57}$. $T_{2}$ mapping is capable of evaluating the condition of articular cartilage in orthopaedics ${ }^{58}$. Although primarily used to evaluate cartilage, intervertebral discs, and bony structures, the biochemical changes, especially water content changes in tendons that predispose or indicate tendinopathy, could be a future target for $T_{2}$ mapping $2,9,10,59-61$.

\section{Conclusion}

Tendon evaluation using ultrasound and magnetic resonance imaging modalities play a crucial role in the identification of normal and abnormal tendon structure. Knowledge of the capabilities and limitations of these methods is important for clinicians regarding diagnosis and prognosis. Clinicians should also be aware of the emerging imaging technologies that may someday assist them to successfully diagnose and treat tendon disease.

\section{References}

1. Birch $\mathrm{HL}$. Tendon matrix composition and turnover in relation to functional requirements. Int J Exp Pathol. 2007;88(4):241248.

2. Hodgson R, O'Connor P, Grainger A. Tendon and ligament imaging. Br J Radiol. 2012;85(1016):1157-1172.

3. Kannus P. Structure of the tendon connective tissue. Scand J Med Sci Sports. 2000;10(6):312-320.

4. Józsa LG, Kannus P. Human tendons: Anatomy, physiology, and pathology. Human Kinetics Champaign, IL; 1997.

5. Tresoldi I, Oliva F, Benvenuto M, et al. Tendon's ultrastructure. Muscles, ligaments and tendons journal. 2013;3(1):2. 
6. D'Agostino MA, Olivieri I. Enthesitis. Best Practice \& Research Clinical Rheumatology. 2006;20(3):473-486.

7. McGonagle D, Marzo-Ortega $\mathrm{H}, \mathrm{O}^{\prime} \mathrm{C}$-onnor $\mathrm{P}$, et al. Histological assessment of the early enthesitis lesion in spondyloarthropathy. Ann Rheum Dis. 2002;61(6):534-537.

8. Andres BM, Murrell GA. Treatment of tendinopathy: What works, what does not, and what is on the horizon. ClinOrthop. 2008;466(7):1539-1554.

9. de Mos M, van El B, DeGroot J, et al. Achilles tendinosis changes in biochemical composition and collagen turnover rate. Am J Sports Med. 2007;35(9):1549-1556.

10. Kannus P, Jozsa L. Histopathological changes preceding spontaneous rupture of a tendon. A controlled study of $891 \mathrm{pa}-$ tients. The Journal of bone and joint surgery.American volume. 1991;73(10):1507-1525.

11. Khan KM, Cook JL, Bonar F, Harcourt P, Åstrom M. Histopathology of common tendinopathies. Sports Medicine. 1999; 27(6):393-408.

12. Riley G, Harrall R, Constant C, Chard M, Cawston T, Hazleman B. Glycosaminoglycans of human rotator cuff tendons: Changes with age and in chronic rotator cuff tendinitis. Ann Rheum Dis. 1994;53(6):367-376.

13. Tuite $D$, Renström $P, O$ 'brien $M$. The aging tendon.Scand $J$ Med Sci Sports. 1997;7(2):72-77.

14. Fenwick SA, Hazleman BL, Riley GP. The vasculature and its role in the damaged and healing tendon. Arthritis research. 2002;4(4):252-260.

15. Shinbane JS, Colletti PM, Shellock FG. Magnetic resonance imaging in patients with cardiac pacemakers: Era of "MR conditional" designs. J Cardiovasc Magn Reson. 2011;13(63):63.

16. GuinelFilho H, Du J, Pak BC, et al. Quantitative characterization of the achilles tendon in cadaveric specimens: $\mathrm{T} 1$ and $\mathrm{T}^{*}$ measurements using ultrashort-TE MRI at $3 \mathrm{~T}$. Am J Roentgenol. 2009;192(3):W117-W124.

17. Gold GE, Wren T, Nayak K, Nishimura D, Beaupre G. In vivo short echo time imaging of achilles tendon. 2001;244.

18. Du J, Chiang AJ, Chung CB, et al. Orientational analysis of the achilles tendon and enthesis using an ultrashort echo time spectroscopic imaging sequence. MagnReson Imaging. 2010;28(2):178-184.

19. Oatridge A, Herlihy AH, Thomas RW, et al. Magnetic resonance: Magic angle imaging of the achilles tendon. The Lancet. 2001;358(9293):1610-1611.

20. Fullerton GD, Rahal A. Collagen structure: The molecular source of the tendon magic angle effect. Journal of Magnetic Resonance Imaging. 2007;25(2):345-361.

21. Peto $S$, Gillis $P$, Henri VP. Structure and dynamics of water in tendon from NMR relaxation measurements. Biophys J. 1990; 57(1):71-84.

22. Magee T, Williams D. 3.0-T MRI of the supraspinatus tendon. Am J Roentgenol. 2006;187(4):881-886.

23. Kamel M, Eid H, Mansour R. Ultrasound detection of heel enthesitis: A comparison with magnetic resonance imaging. J Rheumatol. 2003;30(4):774-778.

24. Goutallier D, Postel J, Bernageau J, Lavau L, Voisin M. Fatty infiltration of disrupted rotator cuff muscles. Rev RhumEngl Ed. 1995;62(6):415-422.

25. Kany J, Flurin P, Richardi G, Hardy P. Rotator cuff tear imaging in patients over 70 years: Specific MRI findings? Orthopaedics\& Traumatology: Surgery \& Research. 2013;99(8, Supplement):S385-S390.

26. Cardinal É, Chhem RK, Beauregard CG.Ultrasound-guided interventional procedures in the musculoskeletal system. RadiolClin North Am. 1998;36(3):597-604.

27. Walz DM, Newman JS, Konin GP, Ross G. Epicondylitis: Pathogenesis, imaging, and treatment1. Radiographics. 2010;30(1):167-184.

28. Miller TT, Shapiro MA, Schultz E, Kalish PE. Comparison of sonography and MRI for diagnosing epicondylitis.Journal of clinical ultrasound. 2002;30(4):193-202.

29. Kim HM, Dahiya N, Teefey SA, et al. Location and initiation of degenerative rotator cuff TearsAn analysis of three hundred and sixty shoulders. The Journal of Bone \& Joint Surgery. 2010;92(5):1088-1096.

30. Via AG, De Cupis M, Spoliti M, Oliva F. Clinical and biological aspects of rotator cuff tears. Muscles, ligaments and tendons journal. 2013;3(2):70.

31. Patten RM. Tears of the anterior portion of the rotator cuff (the subscapularis tendon): MR imaging findings. AJR Am J Roentgenol. 1994;162(2):351-354.

32. Itoi E, Tabata S. Incomplete rotator cuff tears: Results of operative treatment. ClinOrthop. 1992;284:128-135.

33. Reinus WR, Shady KL, Mirowitz SA, Totty WG. MR diagnosis of rotator cuff tears of the shoulder: Value of using T2-weighted fat-saturated images. AJR Am J Roentgenol. 1995; 164(6): 1451-1455.

34. Oliva F, Via AG, Maffulli N. Physiopathology of intratendinous calcific deposition. BMC Med. 2012;10:95-7015-10-95.

35. van Holsbeeck MT, Kolowich PA, Eyler WR, et al. US depiction of partial-thickness tear of the rotator cuff. Radiology. 1995;197(2):443-446.

36. de Jesus JO, Parker L, Frangos AJ, Nazarian LN. Accuracy of MRI, MR arthrography, and ultrasound in the diagnosis of rotator cuff tears: A meta-analysis. Am J Roentgenol. 2009; 192 (6):1701-1707.

37. Westacott DJ, Minns JI, Foguet P. The diagnostic accuracy of magnetic resonance imaging and ultrasonography in gluteal tendon tears--a systematic review. Hip Int. 2011;21(6):637645.

38. Lequesne M, Djian P, Vuillemin V, Mathieu P. Prospective study of refractory greater trochanter pain syndrome. MRI findings of gluteal tendon tears seen at surgery.clinical and MRI results of tendon repair. Joint Bone Spine. 2008;75(4):458-464.

39. Kingzett-Taylor A, Tirman PF, Feller J, et al. Tendinosis and tears of gluteus medius and minimus muscles as a cause of hip pain: MR imaging findings. AJR Am J Roentgenol. 1999;173(4):1123-1126.

40. Kong A, Van der Vliet A, Zadow S. MRI and US of gluteal tendinopathy in greater trochanteric pain syndrome. EurRadiol. 2007;17(7):1772-1783.

41. Reiff DB, Heenan SD, Heron CW. MRI appearances of the asymptomatic patellar tendon on gradient echo imaging. Skeletal Radiol. 1995;24(2):123-126.

42. Cook J, Khan KM, Kiss Z, Coleman B, Griffiths L. Asymptomatic hypoechoic regions on patellar tendon ultrasound: $A$ 4-year clinical and ultrasound followup of 46 tendons. Scand J Med Sci Sports. 2001;11(6):321-327.

43. Warden SJ, Kiss ZS, Malara FA, Ooi AB, Cook JL, Crossley $\mathrm{KM}$. Comparative accuracy of magnetic resonance imaging and ultrasonography in confirming clinically diagnosed patellar tendinopathy.Am J Sports Med. 2007;35(3):427-436.

44. Padulo J, Oliva F, Frizziero A, Maffulli N. Muscle, Ligaments and Tendons Journal. Basic principles and recommendations in clinical and field science research. MLTJ. 2013;4:250-252.

45. Soila K, Karjalainen PT, Aronen HJ, Pihlajamaki HK, Tirman PJ. High-resolution MR imaging of the asymptomatic achilles tendon: New observations. AJR Am J Roentgenol. 1999; 173 (2):323-328.

46. Maffulli N, Regine R, Angelillo M, Capasso G, Filice S. Ultrasound diagnosis of achilles tendon pathology in runners. $\mathrm{Br} J$ Sports Med. 1987;21(4):158-162.

47. Calleja M, Connell DA. The achilles tendon. 2010;14(03):307322.

48. Gibbon W, Cooper J, Radcliffe G. Sonographic incidence of tendon microtears in athletes with chronic achillestendinosis. Br J Sports Med. 1999;33(2):129-130. 
49. Abate M, Oliva F, Schiavone C, Salini V. Achilles tendinopathy in amateur runners: Role of adiposity (tendinopathies and obesity). Muscles, Ligaments and Tendons Journal. 2012;2 (1):44.

50. Kayser R, Mahlfeld K, Heyde C. Partial rupture of the proximal achilles tendon: A differential diagnostic problem in ultrasound imaging. Br J Sports Med. 2005;39(11):838-842.

51. Tyler DJ, Robson MD, Henkelman RM, Young IR, Bydder GM. Magnetic resonance imaging with ultrashort TE (UTE) PULSE sequences: Technical considerations. Journal of Magnetic Resonance Imaging. 2007;25(2):279-289.

52. Itoh A, Ueno E, Tohno E, et al. Breast disease: Clinical application of US elastography for Diagnosis1. Radiology. 2006; 239(2):341-350.

53. Hall TJ. AAPM/RSNA physics tutorial for residents: Topics in US beyond the basics: Elasticity imaging with US1. Radiographics. 2003;23(6):1657-1671.

54. Khoury V, Cardinal É. "Tenomalacia": A new sonographic sign of tendinopathy? EurRadiol. 2009;19(1):144-146.

55. Garra BS. Imaging and estimation of tissue elasticity by ultrasound.Ultrasound quarterly. 2007;23(4):255-268.
56. Gray ML, Burstein D, Xia Y. Biochemical (and functional) imaging of articular cartilage. 2001;5(04):329-344.

57. Mosher TJ, Dardzinski BJ. Cartilage MRI T2 relaxation time mapping: Overview and applications. 2004;8(04):355-368.

58. Stahl R, Blumenkrantz G, Carballido-Gamio J, et al. MRI-derived $\mathrm{T} 2$ relaxation times and cartilage morphometry of the tibio-femoral joint in subjects with and without osteoarthritis during a 1-year follow-up. Osteoarthritis and Cartilage. 2007;15(11):1225-1234.

59. Nikkuni $Y$, Nishiyama $H$, Hayashi $T$. Clinical significance of $T 2$ mapping MRI for the evaluation of masseter muscle pain in patients with temporomandibular joint disorders. Oral Radiology. 2013;29(1):50-55.

60. Stelzeneder D, Welsch GH, Kovács BK, et al. Quantitative T2 evaluation at 3.0 T compared to morphological grading of the lumbar intervertebral disc: A standardized evaluation approach in patients with low back pain. Eur J Radiol. 2012;81(2): 324-330.

61. Longwei X. Clinical application of diffusion tensor magnetic resonance imaging in skeletal muscle.Muscles, Ligaments and Tendons Journal. 2012;2(1):19. 